Supporting Information

\title{
Untethered Single Cell Grippers for Active Biopsy
}

Qianru Jin, ${ }^{1, \forall}$ Yuqian Yang, ${ }^{1}$ Julian A. Jackson, ${ }^{1}$ ChangKyu Yoon, ${ }^{2, ~ \# ~ D a v i d ~ H . ~ G r a c i a s ~}{ }^{1,2, ~ *}$

${ }^{1}$ Department of Chemical and Biomolecular Engineering, Johns Hopkins University, Baltimore, Maryland 21218, United States

${ }^{2}$ Department of Materials Science and Engineering, Johns Hopkins University, Baltimore, Maryland 21218, United States

${ }^{\forall}$ Present address: Disease Biophysics Group, John A. Paulson School of Engineering and Applied Sciences, Harvard University, Cambridge, MA 02138, USA

\#Present address: Department of Mechanical Systems Engineering, Sookmyung Women’s University, Seoul, 04310, Republic of Korea 


\section{Supplementary methods and models}

\section{Fabrication process of the single cell grippers}

We fabricated the grippers based on previously reported methods, ${ }^{21,22}$ with the introduction of new functional layers. Briefly, we first deposited a sacrificial layer of copper (Cu) on a bare silicon (Si) wafer; the sacrificial layer facilitates the release of the grippers from the wafer after fabrication. Second, we photopatterned and deposited a bilayer of silicon monoxide and silicon dioxide by e-beam evaporation. The release of energy from this differentially stressed bilayer drives self-folding of the grippers. In this study, we used a bilayer of $10 \mathrm{~nm}$ silicon monoxide (Kurt J Lesker, EVMSIO-1065B) and $20 \mathrm{~nm}$ silicon dioxide (Kurt J Lesker, EVMSIO21-5D) to achieve the desired fold angle. Third, we photopatterned thicker rigid segments composed of $100 \mathrm{~nm}$ silicon dioxide and $100 \mathrm{~nm}$ iron (Kurt J Lesker, EVMFE35QXQD) by ebeam evaporation. Next, we patterned the paraffin (details in the following section). After fabrication, we released the grippers from the substrate by dissolving the $\mathrm{Cu}$ sacrificial layer in a commercial $\mathrm{Cu}$ etchant ammonium persulfate (APS-100, Transene). Prior to their release from the wafer, we rinsed the grippers for three times with PBS to remove residual APS.

\section{Paraffin patterning}

The first two fabrication steps for the stress layer and the rigid segments establish the basic components of the grippers. Next, we patterned paraffin wax as a thermally responsive layer to trigger the folding of grippers on heating. First, we patterned a photoresist mold to define the shape and thickness of the paraffin. We spin coated S1813 photoresist on the silicon wafer at $3000 \mathrm{rpm}$. After soft bake at $115^{\circ} \mathrm{C}$ for 1 min, we aligned a photomask with the wafer using registry marks, so as to expose the resist only in the regions of the grippers to UV light with an energy of $150 \mathrm{~mJ} / \mathrm{cm}^{2}$. We then developed the exposed photoresist in AZ 319 MIF developer to leave behind photoresist only in the regions without the grippers.

We first melted the paraffin wax (Sigma, 411663) at $120^{\circ} \mathrm{C}$. We then placed the patterned wafer on the chuck inside the spin coater, and held a heat gun at a distance and towards the wafer to heat it to approximately $140^{\circ} \mathrm{C}$. We quickly dispensed $1 \mathrm{~mL}$ of liquid wax onto the patterned wafer and started the spin coating cycle while continuing to direct the heat source during spin coating. We spincoated the molten wax at $5000 \mathrm{rpm}$ for $1 \mathrm{~min}$ and then cooled the wafer in air overnight. After wax coating and cooling, we washed the wafer in acetone, and then rinsed the wafer in isopropyl alcohol (IPA) and deionized water to remove the underlying photoresist. After this process, the remaining paraffin wax covers only the region of the grippers and the grippers are ready to be released and used. For butter triggered grippers, we patterned food grade butter on the grippers using a similar method but at a lower temperature.

\section{Microfluidic chamber fabrication, and gripper actuation}

We fabricated microfluidic chambers to investigate guidance of single cell grippers through narrow, bifurcated, and winding channels. We designed the channel patterns in AutoCAD and transferred them to a photomask. Then, we pretreated a clean Si wafer with hexamethyldisilazane (HMDS) and spin coated SU8 2050 at $1700 \mathrm{rpm}$ to create a $100 \mu \mathrm{m}$ thick pattern. After soft baking at $65^{\circ} \mathrm{C}$ for $5 \mathrm{~min}$ and $95^{\circ} \mathrm{C}$ for $15 \mathrm{~min}$, we exposed the wafer to $240 \mathrm{~mJ} / \mathrm{cm}^{2} \mathrm{UV}$ light through the photomask to transfer the pattern from the photomask to the SU8. We post exposure baked the wafer at $65^{\circ} \mathrm{C}$ for $5 \mathrm{~min}$ and $95^{\circ} \mathrm{C}$ for $10 \mathrm{~min}$ and then developed the patterns in SU8 developer for another $10 \mathrm{~min}$. We rinsed the wafer with IPA and DI water, and then hard baked the SU8 resist at $200^{\circ} \mathrm{C}$ for $30 \mathrm{~min}$.

We thoroughly mixed PDMS elastomer and the curing agent (Dow Corning) at a ratio of 10:1 and degassed the solution in a desiccator for at least 2 hours before pouring the mixture onto the pattern. We cured the PDMS at $75^{\circ} \mathrm{C}$ overnight. After curing, we used a blade to cut and peel the PDMS patterns off. We punched holes in the patterned PDMS using a $1.5 \mathrm{~mm}$ portable biopsy punch at designated positions 
and then treated the PDMS interface with an oxygen plasma. We then bonded a glass slide to the PDMS to seal the channels. The microfluidic chamber was then ready to use.

To trigger gripper actuation, we applied heat by one of three methods; either by warming up the solution on a hot plate during imaging with an upright microscope, or by directing heat with a heat gun during imaging on an inverted microscope or using a temperature-controlled chamber in a confocal microscope. When we used a hot plate, we added the cells and released grippers in PBS in a small dish, and placed the dish on a hot plate. We inserted a digital probe into the dish to monitor the temperature change during the gripper folding process. First, at room temperature, we steered the gripper towards the targeted cells using a magnetic field. When the gripper reached the desired location, we turned on the hot plate to a pre-calibrated setting to increase the temperature. For fast actuation and high-resolution imaging under inverted microscope, we used a heat gun to apply heat remotely and used the digital temperature probe to monitor the temperature. For live cell imaging in the single cell grippers we heated the solution using a temperature-controlled chamber. In this study, we estimate the gripper trigger temperature to be $37 \pm 2^{\circ} \mathrm{C}$ and attribute the standard deviation to variations in the paraffin wax thickness. For in vivo applications which require remote heat delivery, we envision methods such as induction heating or high-intensity focused ultrasound to selectively heat the iron-containing grippers to deliver the heat specifically and minimize collateral damage.

\section{Cell culture and cell cluster preparation}

We maintained WI-38 human fetal lung fibroblasts in Minimal Essential Medium (Corning Cellgro, 10-010-CV) supplemented with 10\% fetal bovine serum (FBS; Thermo Fisher, 16140071), 1\% penicillinstreptomycin (Thermo Fisher, 15140122), 1\% sodium pyruvate (MilliporeSigma, S8636) and 1\% nonessential amino acids (MilliporeSigma, M7145). We maintained the breast cancer cell line MDA-MB-231 in DMEM with 10\% FBS and 1\% penicillin-streptomycin.

We cultured fibroblasts until a desired confluence was reached (80\%). After removing the culture media, we added $1 \mathrm{~mL}$ trypsin to retrieve the adherent cells. We then centrifuged the cell suspension at 1000 rpm for $3 \mathrm{~min}$. Instead of resuspending the cells, we extracted $10 \mu \mathrm{L}$ of the mixture from the bottom of the tube and used it as the cell cluster in our cell excision experiments.

\section{Cell staining, fluorescence imaging and live/dead assay}

We permeabilized the cells using $0.1 \%$ Triton-X in a $4 \%$ paraformaldehyde (PFA) solution for 2 min and fixed the cells using a 4\% PFA solution for $20 \mathrm{~min}$. We stained the cells for $\beta$-tubulin using antibody (Abcam, ab6046, 1:100 dilution) and Alexa Fluor 488 (Jackson ImmunoResearch, 111-545-003, 1:100 dilution), and nuclei using 0.1\% DAPI (Thermo Fisher, 62248).

For the live/dead assay during the gripper closing process, we first rinsed and trypsinized the adherent cells, and collected and incubated them with PBS containing $1 \mu \mathrm{M}$ calcein AM and $2 \mu \mathrm{M}$ ethidium homodimer- 1 for 30 minutes. Next, we added about $200 \mu \mathrm{L}$ cell solution to a small dish for imaging and a droplet containing the released grippers in PBS to the solution. We placed the dish in a temperaturecontrolled chamber for simultaneous confocal fluorescence microscopy and gripper actuation. We triggered the gripper closing by increasing the temperature in the chamber from room temperature to $37^{\circ} \mathrm{C}$ using the controlled heating blocks in the chamber. We monitored the fluorescence signals from the cells continuously throughout and after the gripper closing process.

\section{Ex-vivo tissue preparation}

We purchased tissue microscope slides from Carolina Biological Supply Company (Item \# 316102). We disassembled the slides in order to remove the coverslip and to allow accessibility of the grippers to the tissue. Once we removed the coverslip, we added a drop of gripper-containing solution onto the tissue.

\section{Dynamic Mechanical Analysis (DMA)}


We molded and used $\sim 1 \mathrm{~cm}^{3}$ paraffin cubes for DMA measurements. To obtain elastic moduli values for the wax at different temperatures, we stabilized the chamber temperature for more than 30 min to reach a steady temperature. We subjected the samples to an unconfined compression, controlled force deformation test (Q800 DMA, TA instruments). We increased the applied force at a rate of $2 \mathrm{~N} / \mathrm{min}$ until a maximum static force of $2 \mathrm{~N}$, after which we reduced the load at a rate of $4 \mathrm{~N} / \mathrm{min}$ to $0 \mathrm{~N}$.

\section{Fold angle analysis by analytical solution and finite element analysis}

We modeled the fold angle of the grippers using an analytical solution adopted from the literature, ${ }^{32}$ and finite element analysis. We considered the hinge to be composed of three layers: silicon monoxide, silicon dioxide and paraffin. We estimated the differential strain in the bilayer to be 0.0043 based on a previous study. ${ }^{21}$ The material properties used are listed below in the format as: material (Young's modulus, Poisson's ratio, thickness). Silicon monoxide (77 GPa, 0.2, $10 \mathrm{~nm}$ ), silicon dioxide (75 GPa, 0.17, $20 \mathrm{~nm}$ ), cold paraffin (6.44 MPa, 0.2, variable) and warm paraffin (0.37 MPa, 0.2, variable).

For finite element analysis (FEA), we used a shell model to construct the gripper, due to the large ratio between the lateral dimensions and the thickness. We adopted the model from a prior publication. ${ }^{21}$ We based the gripper dimensions on the AutoCAD design. We defined the thickness of the flexible hinge as described in the previous paragraph. We used a static model and considered non-linear deformation to capture the large deformation. We used a mesh size of $2 \mu \mathrm{m}$ and the element type of S4R in the model. We performed a convergence study to verify that a further increase of mesh numbers did not change the results. We set the boundary conditions as below. 1: We fixed the $x$-axis and $y$-axis at the center of the gripper to be $d x=d y=d z=0 ; 2$ : We considered the $x$-axis and y-axis throughout the gripper to be $y$-symmetrical and $\mathrm{x}$-symmetrical. We assigned a thermal expansion coefficient to the silicon monoxide layer and applied a temperature field only at the hinge, to simulate the differential stress between the bilayer. We used a strain difference of 0.0043 based on the previous measurement. ${ }^{21}$

\section{Estimation of the magnetic field gradient to move the single cell grippers}

We used an analytical model to estimate the magnetic field gradient that is needed to move the single cell grippers in a fluid environment. We based the analysis on a model from a previous study. ${ }^{33}$

If we assume that the micro-object is suspended in liquid without friction with solid surface, we can write the translational equations of motion in the planar workspace as:

$$
F_{e m}+F_{d}+F_{i}=0
$$

where $F_{e m}$ is the electromagnetic force, $F_{d}$ is the drag force, and $F_{i}$ is the inertial force. In the case of single cell grippers, where speed $v=100 \mu \mathrm{m} / \mathrm{s}$, feature length $L=70 \mu \mathrm{m}$, water density $\rho=1 \times 10^{3} \mathrm{~kg} / \mathrm{m}^{3}$, and water viscosity $\mu=8.90 \times 10^{-4} \mathrm{~Pa} \cdot s$ at $25^{\circ} \mathrm{C}$, the Reynolds number,

$$
R e=\rho \frac{L v}{\mu}=7.86 \times 10^{-3}<0.01
$$

When the Reynolds number $R e<0.01$, we can neglect the inertial force relative to the other forces. With this assumption that $F_{i}$ is negligible, equation (1) becomes,

$$
F_{e m}+F_{d}=0
$$

Since the grippers move at sub-centimeter speeds in a flow-less environment, we can write an equation for $F_{d}$ as,

$$
F_{d}=\frac{1}{2} \rho C_{D} A v^{2}=2 \times 10^{-13} N
$$

where the fluid density $\rho=1 \times 10^{3} \mathrm{~kg} / \mathrm{m}^{3}$, cross sectional area normal to the direction of motion $A=$ $82 \mu \mathrm{m}^{2}$ and the speed of the gripper $v=100 \mu \mathrm{m} / \mathrm{s}$. We can estimate the drag coefficient $C_{D}$ to be $C_{D}=$ 500 based on prior studies of the drag coefficient of a plate in a parallel flow at $\operatorname{Re}=0.01 .^{35}$ 
Considering the unidirectional motion of gripper, we can write an equation for the electromagnetic force in the one-dimensional magnetic gradient field as,

$$
F_{e m}=\nabla(m \cdot B)=m \cdot \nabla \mathrm{B}
$$

We can also estimate the magnetic dipole moment $m$ as,

$$
m=M_{s} \cdot V=1.2 \times 10^{-10} A \cdot m^{2}
$$

where the iron material volume $V=7 \times 10^{-17} \mathrm{~m}^{3}$, saturation magnetization $M_{S}$ for the approximately 100 nm thick iron thin film is $M_{S}=1.7 \times 10^{6} \mathrm{~A} / \mathrm{m}^{36}$

be,

Hence, we can estimate the required magnetic field gradient $\nabla \mathrm{B}$ to move the single cell gripper to

$$
\nabla \mathrm{B}=-\frac{F_{d}}{m}=-1.6 \times 10^{-3} \mathrm{~T} / \mathrm{m}
$$


Supplementary figures
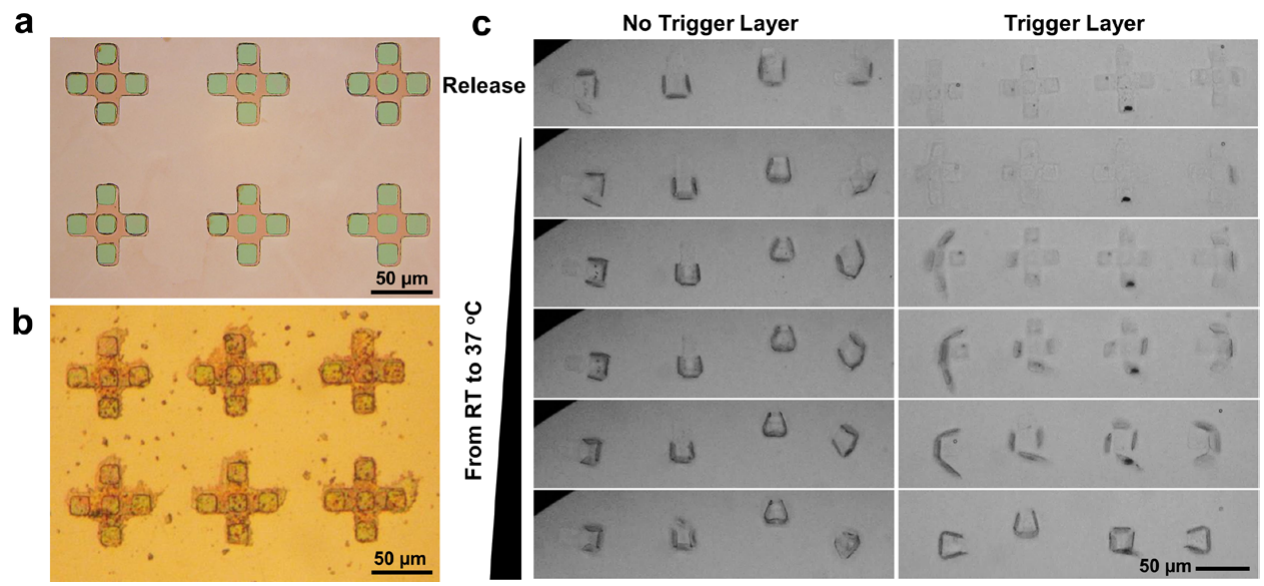

Figure S1. Butter as the trigger layer for thermal actuation of single cell grippers. (a) Optical image of a control sample containing an array of grippers with square arms fabricated without a butter coating. The arms contain $100 \mathrm{~nm}$ of silicon dioxide and $100 \mathrm{~nm}$ of iron. (b) Optical image of an array of grippers coated with a thin layer of butter on top of the grippers. (c) Optical images showing the folding process of grippers without butter (left), and with butter (right). After release, grippers without the trigger layer closed immediately, while grippers with trigger layer stayed open. As the temperature was increased from room temperature to $37^{\circ} \mathrm{C}$, the grippers with the butter trigger layer started to close, when the butter softened. 


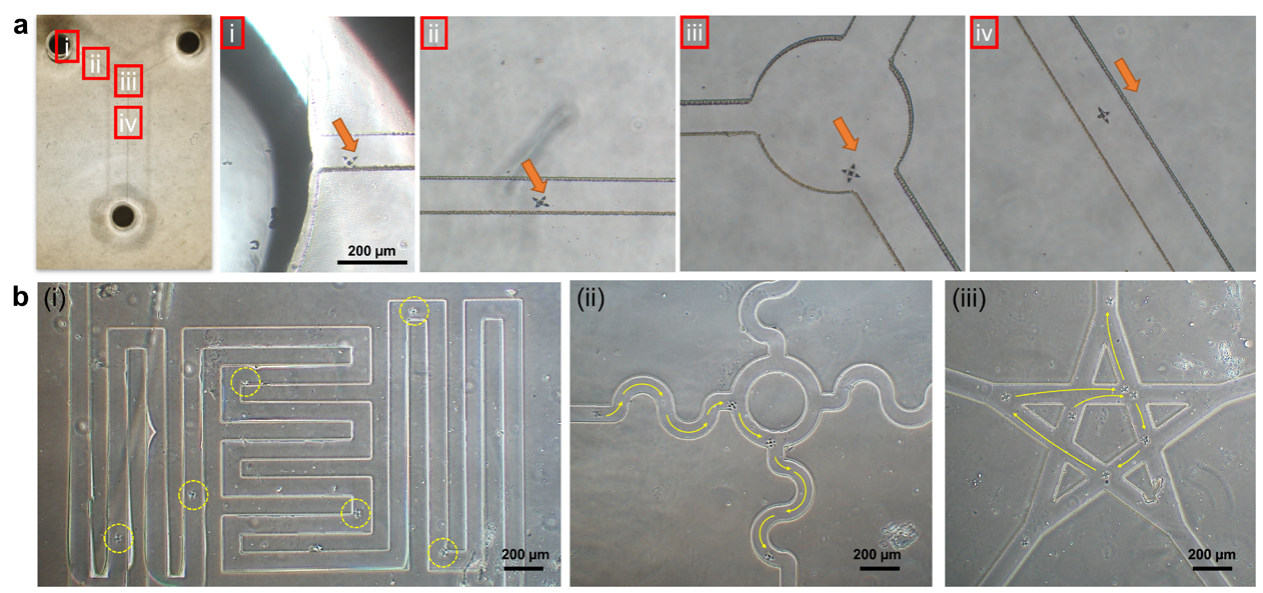

Figure S2. Grippers moving in narrow, bifurcated, and winding microfluidic channels guided by a magnetic field. (a) Optical images of a gripper navigated through a microfluidic channel with bifurcations. An overview of the microfluidic channel followed by details of the gripper: (i) leaving the initial chamber, (ii) moving through the channel, (iii) reaching the mixing chamber, (iv) exiting the mixing chamber. (b) Optical images of grippers being navigated through microfluidic channels of different configurations. The images show the motion path of a gripper at multiple time points, in, (i) a zig-zag channel, (ii) a twisted channel, and (iii) a star-shaped channel with many exits. 

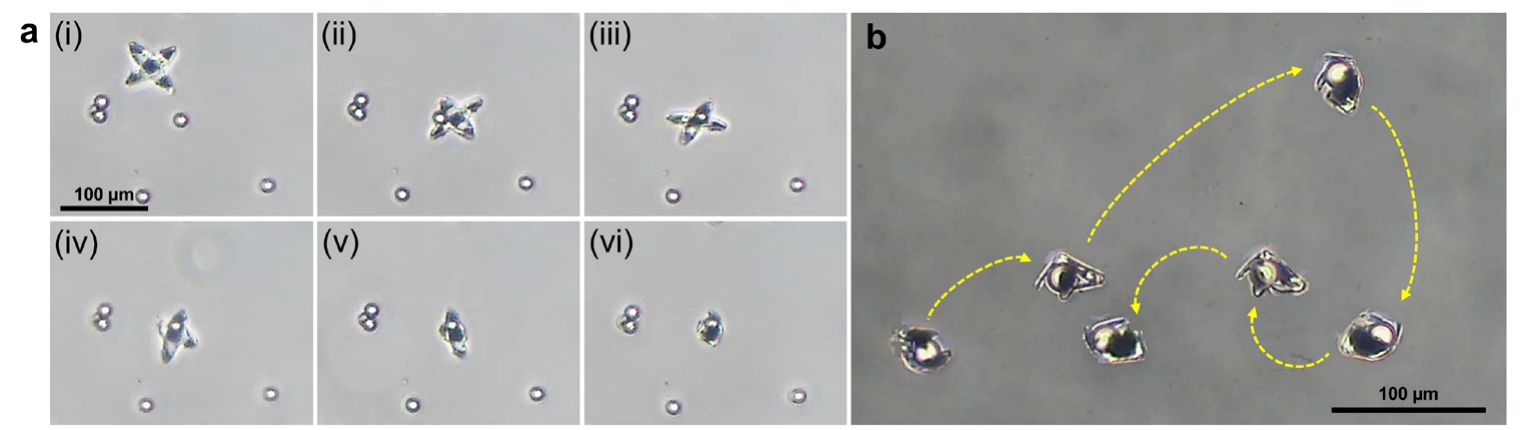

Figure S3. Grippers capturing microbeads by magnetic guidance and thermal actuation. (a) (i-iii) Optical image sequence showing a gripper approaching a polystyrene microbead ( $15 \mu \mathrm{m}$ diameter) from a distance guided by the magnetic field; (iv-vi) The gripper started closing and captured the bead upon temperature increase. (b) Optical image sequence showing a gripper carrying the microbead guided by the magnetic field and navigating through an arbitrary trace. 


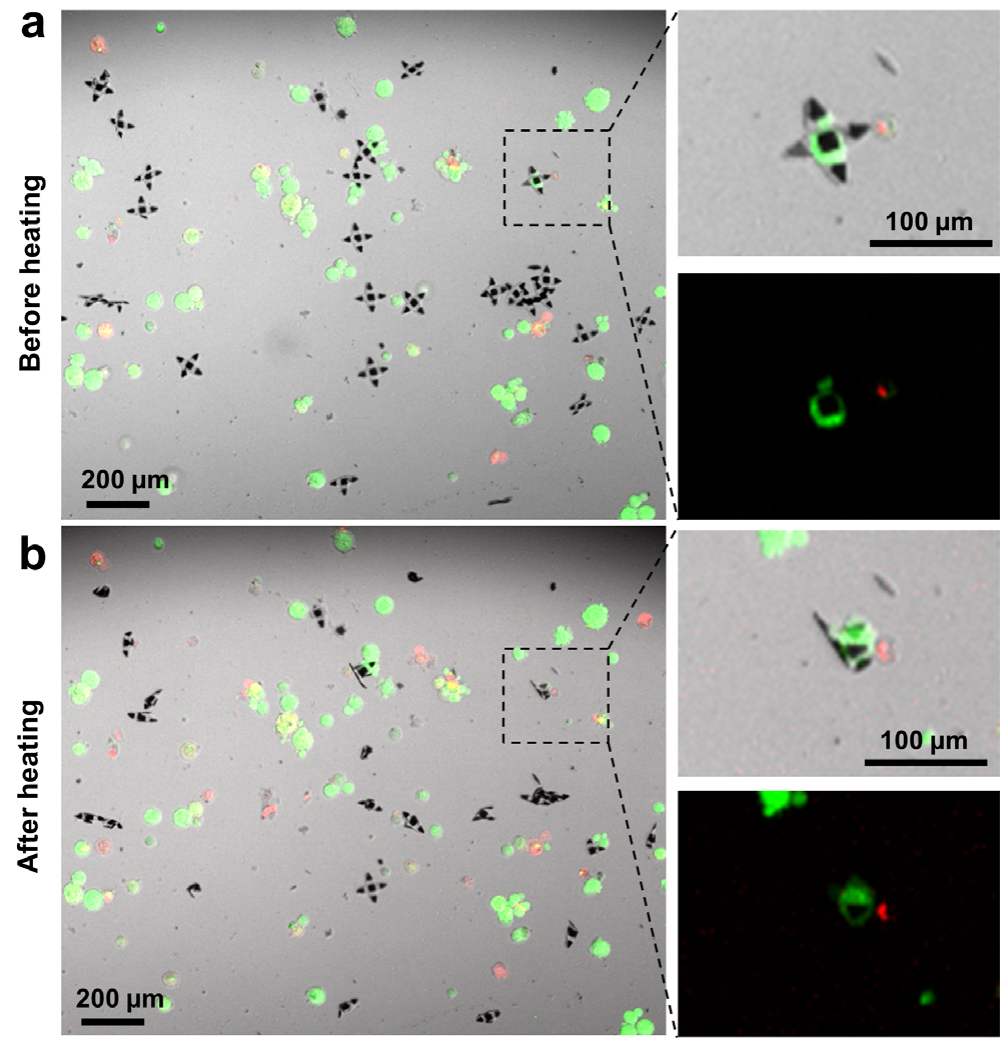

Figure S4. Biocompatibility of the self-folding process. (a) Optical fluorescence microscopy images showing grippers and cells that were stained by calcein AM and ethidium homodimer for a live/dead assay. The green fluorescence indicates live cells and red color indicates dead cells. (b) Optical fluorescence microscopy images showing that a majority of cells remained alive after temperature increase from room temperature to $37^{\circ} \mathrm{C}$. A few cells with red fluorescence were from the sediment of suspended dead cells. The right zoom-in images show a gripper capturing a single cell. The time interval between the acquisition of the two images was approximately 30 minutes, with calcein AM and ethidium homodimer present in the solution throughout the process. 

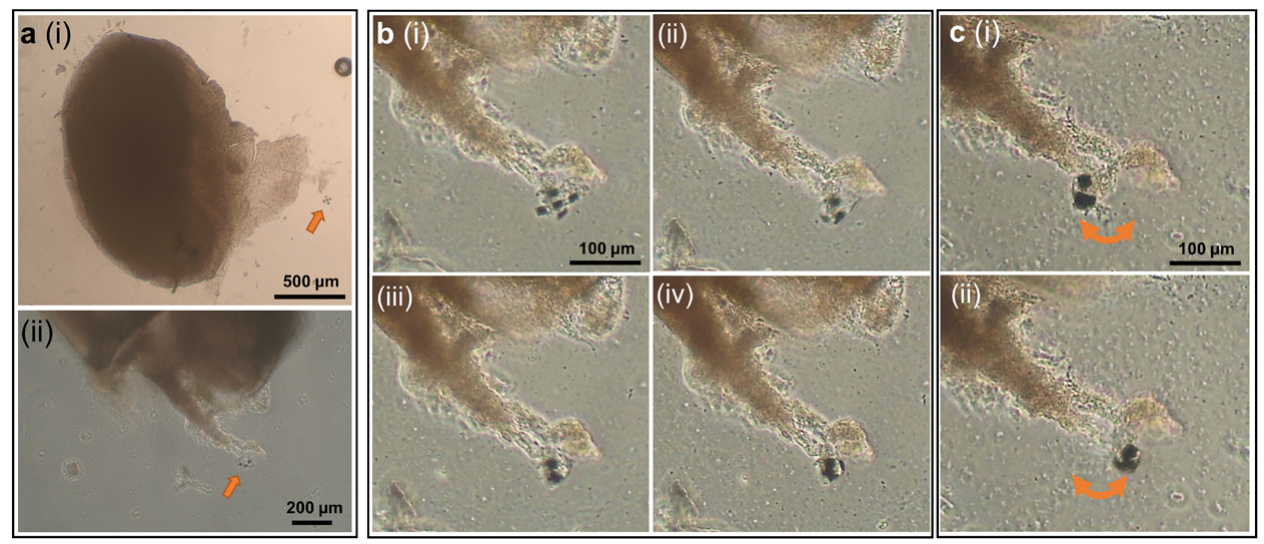

Figure S5. Grippers pulling liver tissue by magnetic locomotion and thermal actuation. (a) Optical image of a gripper approaching a piece of chicken liver tissue from a remote distance and reaching its periphery, guided by a magnetic field. (b) Optical image sequence showing the gripper folding and holding onto a piece of tissue upon temperature increase. (c) Optical image sequence showing that the gripper firmly grasped the tissue and generated large dislocation with strong dragging and twisting motion, steered by the magnetic field. 


\section{References}

21. Malachowski, K.; Jamal, M.; Jin, Q.; Polat, B.; Morris, C. J.; Gracias, D. H. Self-folding single cell grippers. Nano Lett. 2014, 14, 4164-4170.

22. Jin, Q.; Li, M.; Polat, B.; Paidi, S. K.; Dai, A.; Zhang, A.; Pagaduan, J. V.; Barman, I.; Gracias, D. H. Mechanical Trap Surface-Enhanced Raman Spectroscopy for Three-Dimensional Surface Molecular Imaging of Single Live Cells. Angew. Chem., Int. Ed. 2017, 56, 3822-3826.

32. Nikishkov, G. P. Curvature estimation for multilayer hinged structures with initial strains. J. Appl. Phys. 2003, 94, 5333-5336.

33. Ongaro, F.; Scheggi, S.; Ghosh, A.; Denasi, A.; Gracias, D. H.; Misra, S. Design, characterization and control of thermally-responsive and magnetically-actuated micro-grippers at the air-water interface. PLoS One 2017, 12, e0187441.

35. Jones, A.; Knudsen, J. G. Drag coefficients at low Reynolds numbers for flow past immersed bodies. AIChE J. 1961, 7, 20-25.

36. Utsushikawa, Y.; Niizuma, K. The saturation magnetization of Fe-N films prepared by nitriding treatment in N2 plasma. J. Alloys Compd. 1995, 222, 188-192. 